\title{
Rapid profiling of animal-derived fatty acids using fast GC x GC coupled to time-of- flight mass spectrometry
}

\begin{abstract}
Rapid profiling of fatty acid methyl esters (FAME) from five different animal sources was examined in this study using fast comprehensive two-dimensional gas chromatography coupled to time-of-flight mass spectrometry (fast GC x GC-TOFMS). The result showed that GC x GC analysis combining two different microbore columns (SLB-5 ms, $10 \mathrm{~m}$ x $0.10 \mathrm{~mm}$ x $0.10 \mu \mathrm{m}$ and DBWax, $0.5 \mathrm{~m} \times 0.10 \mathrm{~mm} \times 0.10 \mu \mathrm{m}$ ) allowed three-fold faster analysis times than those observed for conventional GC x GC analysis. The modulation ratio (M R) of the system was defined at the value of 2.38 by optimizing the modulation period and offset temperature for precise mass spectral identification and highest effluent resolution in the analysis. In accordance with the normalized FAME level obtained from various animal fats, namely lard (LA), chicken fat (CF), beef tallow (BF), mutton tallow (MF) and cod liver oil (CLO), a clear discrimination of LA from the other species by principal components analysis (PCA) was observed. This was attributed to several FAME constituents involving methyl 6,9,12,15-heneicosatetraenoate (C21:4n-6), methyl 11,14-eicosadienoate (C20:2n-6), trans9,12-methyl octadecadienoate (C18:2n-6t), trans-9-methyl octadecenoate (C18:1n-9t) and methyl hexadecanoate (C16:0).
\end{abstract}

Keyword: GC X GC; TOFMS; Fast GC; FAME; PCA; Animal fats; Lards. 